\title{
Commentaire
}

\section{Surveillance de la maltraitance des enfants : recension, suivi, évaluation et aperçu}

\author{
Rebecca T. Leeb, Ph. D. (1)*; John D. Fluke, Ph. D. (2)
}

Diffuser cet article sur Twitter

La surveillance de la santé publique est [traduction] « la collecte, l'analyse et l'interprétation systématiques et permanentes de données axées sur les résultats en vue de leur utilisation pour la planification, la mise en œuvre et l'évaluation des pratiques en matière de santé publique, étroitement liées à la diffusion opportune de ces données aux personnes qui en ont besoin ${ }^{1(p . ~ 164)}$. Les systèmes de surveillance à long terme sont donc généralement conçus en fonction de trois objectifs fonctionnels : recension, suivi et évaluation. Ce système, conçu au départ dans le contexte de la prévention et du contrôle des maladies infectieuses, a ensuite été appliqué à d'autres problèmes de santé (dont la violence) ayant des répercussions sur les collectivités et la sociétée ${ }^{2,3}$.

Exercer une surveillance épidémiologique sur la violence et la négligence envers les enfants présente des difficultés spécifiques liées à la variabilité des définitions et des modalités dans le rapport des cas. Les définitions de la violence et de la négligence diffèrent entre les pays et en leur sein, empêchant une estimation exacte de l'ampleur du problème. Les définitions varient également en fonction de la nature du système de protection des enfants ${ }^{4}$. Certains pays ne disposent pas de système judiciaire ou social doté de responsabilités précises pour réagir aux cas de violence et de négligence envers les enfants et en consigner les rapports, ce qui est en particulier le cas des pays où certaines populations sont isolées ou en migration (p. ex. en raison de conflits). La sous-déclaration des cas de violence et de négligence envers les enfants entraîne des sous-estimations de la prévalence. Les sévices subis par les enfants ne sont souvent connus que de l'agresseur et, selon le niveau de développement de l'enfant, de sa victime. De plus, les cas de violence et de négligence envers les enfants sont susceptibles d'être signalés à divers intervenants (éducateurs, clergé, médecins, services de police, aide sociale à l'enfance) comme il peuvent ne l'être à aucune source officielle. La stigmatisation sociale et les conséquences non prévisibles des signalements, tout comme les barrières culturelles et politiques, ont aussi des répercussions sur le signalement, tant au sein d'une collectivité qu'à l'échelle planétaire.

Malgré ces difficultés, des systèmes de surveillance épidémiologique des cas de violence et de négligence envers les enfants existent dans la majorité des pays à revenus élevés ainsi que dans un nombre croissant de pays à faibles et moyens revenus. Dans les pays à revenus élevés, la surveillance mise généralement sur les données recueillies auprès d'agences de protection de l'enfance ou de professionnels en contact avec les enfants. Par exemple, l'Étude canadienne sur l'incidence des signalements de cas de violence et de négligence envers les enfants (ECI) dont on traite dans ce numéro spécial recueille les données sur la violence et la négligence envers les enfants fournies par les agences de protection de l'enfance. Elles sont recueillies périodiquement au moyen d'enquêtes et elles sont analysées pour évaluer les taux d'incidence. D'autres programmes de collecte de données usant de méthodes d'enquête sur échantillon similaires, comme la National Incidence Study of Child Abuse and Neglect (NIS) des ÉtatsUnis, obtiennent leurs données des agences de protection de l'enfance, mais aussi d'autres professionnels en contact avec les enfants ${ }^{5}$.

Le National Child Abuse and Neglect Data System (NCANDS) des États-Unis renferme, comme l'ECI, des données sur la protection de l'enfance, mais il fait appel à une autre méthodologie. Les États et territoires fournissent, pour l'analyse des prévalences et des tendances annuelles, des données administratives sur les enfants ayant fait l'objet d'un signalement auprès du système d'aide sociale en raison de violence ou de négligence soupçonnées. Des systèmes utilisant de manière similaire des données administratives obtenues auprès d'agences de protection de l'enfance sont utilisés ailleurs, notamment - pour n'en nommer que quelques-uns - au Royaume-Uni ${ }^{6}$ et en Australie $^{7}$. L'Arabie saoudite a élaboré un système de surveillance qui fait appel à des équipes de protection de l'enfance en milieu hospitalier plutôt qu'à des données fournies par la protection de l'enfance ${ }^{4}$.

Dans les pays à faibles et moyens revenus, particulièrement dans les pays où l'infrastructure en services sociaux est limitée, la surveillance des cas de violence et de négligence envers les enfants mise généralement sur des enquêtes où on interroge les enfants et leurs

Rattachement des auteurs :

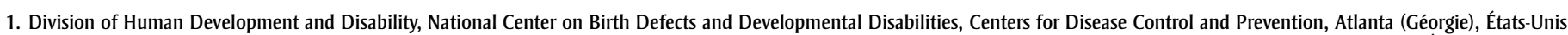
2. Department of Pediatrics, Kempe Center for the Prevention and Treatment of Child Abuse and Neglect, University of Colorado School of Medicine, Aurora (Colorado), États-Unis

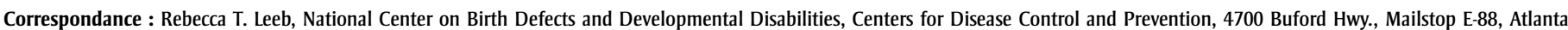
(Géorgie), États-Unis, 30341-3717; tél. : 404-498-6752; téléc. : 404-498-0270; courriel : RLeeb@CDC.gov

* Les constatations et les conclusions présentées dans ce texte sont celles des auteurs et ne reflètent pas la position officielle des Centers for Disease Control and Prevention. 
pourvoyeurs de soins sur leurs expériences actuelles et passées de violence et de négligence dans ce domaine. Citons par exemple les enquêtes Violence Against Children Surveys (VACS) mises en œuvre dans des pays comme le Cambodge, Haïti, le Kenya, le Malawi, le Swaziland, la Tanzanie et le Zimbabwe ${ }^{8}$ et l'Enquête à indicateurs multiples (MICS) de l'UNICEF, mise en œuvre dans plus de 100 pays à faibles et moyens revenus ${ }^{9}$. Notons au passage que ce type d'enquête d'autoévaluation (comme le National Survey of Children's Exposure to Violence [NatSCEV] des États-Unis ${ }^{10}$ ), est également utilisé dans les pays à revenus élevés, parce que ces enquêtes sont en mesure de fournir un plus large éventail de renseignements et de perspectives sur l'incidence et la prévalence des mauvais traitements que les données administratives ou les échantillons issus d'enquêtes auprès de professionnels en contact avec les enfants. Néanmoins, ces enquêtes sont sujettes à des biais spécifiques.

Le manque d'uniformité dans les définitions de cas et d'approche systématique dans la collecte de données au sein des systèmes de surveillance des cas de violence et de négligence envers les enfants témoigne du fait que les trois objectifs de surveillance fonctionnelle sont sans doute nécessaires mais non suffisants à la bonne compréhension de l'ampleur et de la nature de la violence et de la négligence envers les enfants. Même si des mesures ont été prises pour mettre au point des approches définitionnelles harmonisées pour les sources administratives, offrir, dans des milieux complexes sur le plan de la politique où œuvrent de nombreux intervenants intersectoriels, des définitions acceptées demeure un défi ${ }^{11}$. Par ailleurs, nous pensons, comme d'autres intervenants (p. ex. Thacker), que les systèmes de surveillance des cas de violence et de négligence envers les enfants sont incomplets s'ils n'offrent pas un certain éclairage pertinent sur les méthodes de collecte de données, sur le processus de surveillance ou sur les conditions médicales à risque sous-jacentes ${ }^{12}$.

\section{Éclairage pertinent}

L'éclairage pertinent peut être considéré comme une activité analytique générative qui répercute l'information en vue d'améliorer la surveillance et la prévention. Il peut également être ponctuel : une fois proposé, il conduit à une prise de conscience, documentée ou dupliquée, puis les activités ordinaires de surveillance, soit la recension, le suivi et l'évaluation, reprennent leur cours. Voici quatre exemples d'éclairage pertinent pour la surveillance des cas de violence et de négligence envers les enfants.

1. Les modalités de calcul sont importantes. Se fier à une seule source d'information, par exemple aux cas de signalement aux agences de protection de l'enfance ou encore aux enquêtes d'autoévaluation, n'offre qu'une vision partielle du problème. D’après les données dont nous disposons, une triangulation systématique des sources de données est nécessaire pour réaliser pleinement les objectifs de surveillance fonctionnelle et pour brosser un tableau complet de l'ampleur de la violence et de la négligence envers les enfants ${ }^{13,14}$. L'une de ces méthodes de triangulation est le jumelage des données administratives à des identifiants communs à divers systèmes de service, mais elle nécessite des ajustements pour palier l'absence d'uniformité entre les différentes sources. Le Developmental Pathways Project en Australie occidentale a utilisé cette méthode de triangulation avec succès, ce qui a conduit à des changements notables dans les connaissances et les politiques ${ }^{15,16}$. L'enquête NIS utilise une approche différente à l'égard de la triangulation : des enquêtes périodiques sont réalisées auprès de divers intervenants ${ }^{17}$. Cette approche a permis d'orienter et de cibler les efforts en matière de politiques et de pratiques états-uniennes ${ }^{18,19}$, mais une longue périodicité et un coût de collecte de données élevé rendent difficile la détermination des répercussions directes des données de ces enquêtes sur les politiques et pratiques.

2. Savoir quelles personnes et quels éléments sont recensés est important. Par exemple, le NCANDS fournit dans son rapport annuel à la fois des effectifs uniques et des effectifs multiples. L'effectif unique est le nombre d'enfants victimes de maltraitance comptabilisé au cours d'une année donnée. L'effectif multiple est le nombre de rapports de signalement reçus par l'aide sociale à l'enfance au cours de la même année. L'un fournit le nombre d'enfants maltraités alors que l'autre fournit le nombre d'incidents de violence ou de négligence.

3. Le maintien à long terme des systèmes de surveillance nationale est essentiel et complexe. Sans surveillance régulière et continue, l'éclairage pertinent se limite à quelques instantanés, et les études ponctuelles alimentent dans le meilleur des cas les objectifs stratégiques à court terme seulement. Les systèmes de collecte périodique des données, indépendamment de la méthodologie, améliorent ces instantanés, mais n'ont pas obligatoirement la précision requise pour l'examen des conséquences progressives des changements sur une longue période. Les tentatives de l'UNICEF pour assurer le suivi des indicateurs de danger et d'exploitation des enfants à l'aide du MICS démontrent que la surveillance à long terme de la violence et de la négligence envers les enfants est réalisable partout, malgré d'importantes restrictions en matière de ressources ${ }^{20}$.

4. La cible et la portée d'un système de surveillance influencent la nature de ses données. Les systèmes peuvent cibler la morbidité, la mortalité ou les deux, ce qui est susceptible de conduire à des conclusions différentes à propos de la violence et de la négligence envers les enfants. Le choix des indicateurs et des facteurs de risque et de protection aura des répercussions sur les conclusions que l'on pourra tirer des données. Par exemple, l'analyse des données sur la surveillance des enfants dans le système d'aide sociale a offert un éclairage pertinent sur le risque relatif de récidive de maltraitance vécu par les enfants ayant un handicap et a justifié l'importance, pour la recension, le suivi et l'évaluation, de la collecte de données sur les handicaps et sur d'autres facteurs de risques potentiels ${ }^{21}$.

Ces éclairages pertinents sont conformes aux concepts discutés dans le Updated Guidelines for Evaluating Public Health Surveillance Systems des Centers for Disease Control and Prevention des États-Unis ${ }^{22}$. 
Non seulement les éclairages pertinents issus de la surveillance de la violence et de la négligence envers les enfants peuvent-ils aider à évaluer et à améliorer les systèmes de surveillance, mais ils peuvent aussi servir de catalyseur aux responsables des politiques et des programmes. L'information pertinente, bien interprétée, peut aider à réduire la violence envers les enfants et à promouvoir des relations et des environnements favorables à l'épanouissement des enfants. Comme le mentionne Thacker, [traduction] « si l'information [relative à la surveillance] n'est pas fournie aux personnes qui établissent les politiques et qui mettent en œuvre les programmes, son utilisation se limite son archivage et aux projets universitaires [...] $»^{12}$ (p. 5) . Cela signifie que les intervenants clés doivent avoir accès aux données de surveillance.

La surveillance de la violence et de la négligence envers les enfants demeure un défi pour les épidémiologistes du monde entier. Nous espérons que les éclairages pertinents issus de nos travaux collectifs, dont l'ECI, contribueront à mieux orienter nos mesures de prévention face à ce problème mondial de santé publique.

\section{Références}

1. Thacker SB, Berkleman RL. Public health surveillance in the United States. Epidemiol Rev. 1988;10:164-190.

2. Dahlberg LL, Mercy JA. History of violence as a public health problem. AMA J Ethics. 2009;11(2):167-172.

3. Lee LM, Teutsch SM, Thacker SB, St. Louis ME (dir.). Principles and practice of public health surveillance. Oxford Scholarship Online; 2010. DOI: 10.1093/acprof:oso/978 0193572922.001.0001.

4. AlEissa MA, Fluke JD, Gerbaka B et collab. A commentary on national child maltreatment surveillance systems: examples of progress. Child Abuse Neg. 2009;33(11):809-814.

5. Sedlak AJ, Mettenberg J, Basena M, et al. Fourth National Incidence Study of Child Abuse NIS-4: report to Congress. Washington, DC: Department of Health and Human Services, Administration for Children and Families; 2010.
6. Department for Education (UK). Referrals, assessments and children who were the subject of a child protection plan: children in need census 2010 to 2011 (provisional) [statistical release] [Internet]. London (Royaume-Uni) : Department for Education; 2011 [consulté le 17 juin 2015]. Consultable à la page : https://www.gov.uk/government/ statistics/referrals-assessments-and-childrenwho-were-the-subject-of-a-child-protectionplan-children-in-need-census-2010-to-2011provisional

7. Australian Institute of Health and Welfare (AIHW). Child protection Australia: 2011-12. Child welfare series no. 55. Canberra: AIHW; 2013. [CWS 43].

8. Centers for Disease Control and Prevention. Towards a violence-free generation: Using science to fuel action and end violence against children [Internet]. [consultation le 17 juin 2015]. Consultable à la page : http://www.cdc. gov/violenceprevention/vacs/index.html

9. UNICEF. Enquête à indicateurs multiples [Internet]. UNICEF; 2015 [consultation le 17 juin 2015]. Consultable à partir de la page : http://mics.unicef.org/surveys

10. Finkelhor D, Shattuck A, Turner HA, Hamby SL. Trends in children's exposure to violence, 2003 to 2011. JAMA Pediatr. 2014; 168(6):540-546.

11. Leeb RT, Paulozzi LJ, Melanson C, Simon TR, Arias I. Child maltreatment surveillance: uniform definitions for public health and recommended data elements, version 1.0. Atlanta (Géorgie): Centers for Disease Control and Prevention, National Center for Injury Prevention and Control; 2006.

12. Thacker S. Historical Development. Dans : Lee LM, Teutsch SM, Thacker SB, St Louis ME (dir.). Principles and practice of public health surveillance ( $3^{\mathrm{e}}$ éd). New York: Oxford University Press; 2010. p. 1-17.

13. Gibbs D, Rojas-Smith L, Wetterhall S et collab. Improving identification of child maltreatment fatalities through public health surveillance. J Public Child Welf. 2013;7:1-19.

14. Krug EG, Dahlberg LL, Mercy JA, Zwi AB, Lozano R (dir.), Rapport mondial sur la violence et la santé. Genève (Suisse): Organisation mondiale de la santé; 2002.
15. Government of Western Australia, Department of Health. Data linkage Western Australia: Enabling health and medical research in Western Australia [Internet]. Data Linkage WA; 2015 [consultation le 17 juin 2015]. Consultable à la page : http://www. datalinkage-wa.org/

16. Brook EL, Rosman DL, Holman CDJ, Trutwein B. Summary report: research outputs project, WA data linkage unit (1995-2003) [Internet]. Department of Health, Government of Western Australia; 2005. PDF téléchargeable à partir du lien : http://www. datalinkage-wa.org.au/sites/default/files/ Summary \%20of\%20Research \%200utputs \%20Project.pdf

17. U.S. Department of Health and Human Services. The Fourth National Incidence Study of Child Abuse and Neglect [Internet]. [consultation le 17 juin 2015]. Consultable à partir de la page : https://www. nis4.org/index.htm

18. Daro D. Child abuse prevention: a job half done. Chicago: Chapin Hall at the University of Chicago; 2010.

19. Russell J, Cooper C. Technical assistance brief, the NIS-4: what it all means (and doesn't mean) [Internet]. Reno (NV): National Council of Juvenile and Family Court Judges; 2011. PDF téléchargeable à partir du lien : http://www.ncjfcj.org/sites/ default/files/nis-4\%20technical\%20assistance $\% 20$ brief.pdf

20. Sumner SA, Mercy JA, Saul J et collab. Prevalence of sexual violence against children and use of social services - seven countries, 2007-2013. MMWR. 2015;64(21): 565-569.

21. Fluke J, Shusterman G, Hollinshead D, Yuan YT. Longitudinal analysis of repeated child abuse reporting and victimization: multistate analysis of associated factors. Child Maltreatment. 2008;13(1):76-88.

22. Centers for Disease Control and Prevention. Updated Guidelines for Evaluating Public Health Surveillance Systems [Internet]. MMWR. 2001;50(RR13):1-35. Consultable à la page: http://www.cdc.gov/mmwr/ preview/mmwrhtml/rr5013a1.htm 